\title{
PIK3CA Mutation in HPV-Associated OPSCC Patients Receiving Deintensified Chemoradiation
}

\author{
Brian T. Beaty (1) , Dominic H. Moon, Colette J. Shen, Robert J. Amdur, Jared Weiss, \\ Juneko Grilley-Olson, Shetal Patel, Adam Zanation, Trevor G. Hackman, Brian Thorp, \\ Jeffrey M. Blumberg, Samip N. Patel, Mark C. Weissler, Wendell G. Yarbrough, \\ Nathan C. Sheets, Joel S. Parker, D. Neil Hayes, Karen E. Weck, Lori A. Ramkissoon, \\ William M. Mendenhall, Roi Dagan, Xianming Tan, Gaorav P. Gupta, Bhishamjit S. Chera
}

See the Notes section for the full list of authors' affiliations.

Correspondence to: Bhishamjit Chera, Department of Radiation Oncology, University of North Carolina Hospitals, 101 Manning Dr, Campus Box 7512, Chapel Hill, NC 27599-7512 (e-mail: bchera@med.unc.edu).

\begin{abstract}
PIK3CA is the most frequently mutated gene in human papillomavirus (HPV)-associated oropharyngeal squamous cell carcinoma (OPSCC). Prognostic implications of such mutations remain unknown. We sought to elucidate the clinical significance of PIK3CA mutations in HPV-associated OPSCC patients treated with definitive chemoradiation (CRT). Seventyseven patients with HPV-associated OPSCC were enrolled on two phase II clinical trials of deintensified CRT (60 Gy intensitymodulated radiotherapy with concurrent weekly cisplatin). Targeted next-generation sequencing was performed. Of the 77 patients, nine had disease recurrence (two regional, four distant, three regional and distant). Thirty-four patients had mutation(s) identified; 16 had PIK3CA mutations. Patients with wild-type-PIK3CA had statistically significantly higher 3-year disease-free survival than PIK3CA-mutant patients $(93.4 \%, 95 \%$ confidence interval $[\mathrm{CI}]=85.0 \%$ to $99.9 \%$ vs $68.8 \%, 95 \% \mathrm{CI}=$ $26.7 \%$ to $89.8 \% ; P=.004)$. On multivariate analysis, PIK3CA mutation was the only variable statistically significantly associated with disease recurrence (hazard ratio $=5.71,95 \% \mathrm{CI}=1.53$ to $21.3 ; \mathrm{P}=.01$ ). PIK3CA mutation is associated with worse diseasefree survival in a prospective cohort of newly diagnosed HPV-associated OPSCC patients treated with deintensified CRT.
\end{abstract}

Human papillomavirus (HPV)-associated oropharyngeal squamous cell carcinoma (OPSCC) has substantially better prognosis after curative-intent therapy than HPV-negative OPSCC (1). Thus, deintensified therapy for HPV-associated OPSCC patients is being actively investigated (2-4), with a goal of reducing treatment-associated morbidity while maintaining excellent cancer control rates. We have been evaluating a deintensified chemoradiation (CRT) regimen (60 Gy intensity-modulated radiotherapy with concurrent weekly cisplatin) in multiple phase II clinical trials. Disease control is excellent, with 3-year locoregional control of $100 \%(3,5)$. Although p16 status and tobacco pack-years allow for prognostication (1), they are not perfect. Approximately 10-20\% of HPV-associated OPSCC patients develop a recurrence.

We used tumor sequencing results from two deintensification trials to identify mutations that may predict for disease recurrence. Phosphoinositide-3-kinase is an important oncogene, and mutations in its $\mathrm{p} 110 \alpha$ subunit (PIK3CA) have been associated with adverse outcomes in cervical squamous cell carcinoma (6). Their prognostic significance in OPSCC is unclear. Although HPV-associated head and neck squamous cell carcinoma tends to have fewer mutations than HPV-negative disease (7), PIK3CA is commonly mutated in HPVassociated OPSCC $(8,9)$. We sought to elucidate the clinical 
significance of PIK3CA mutations in HPV-associated OPSCC patients treated with definitive CRT.

Seventy-seven patients with HPV-associated OPSCC were enrolled on two phase II clinical trials of deintensified CRT (NCT02281955/NCT03077243) at Universities of North Carolina and Florida. Inclusion criteria were as follows: age 18 years and older, ECOG0-1, T0-3, N0-2, MO OPSCC [AJCC-8 staging (10)], and p16-/HPV-positive (HPV-associated). Patients received intensitymodulated radiotherapy (total $60 \mathrm{~Gy}, 2 \mathrm{~Gy} /$ fraction). Patients with TP53 mutations received $70 \mathrm{~Gy}$. Concurrent chemotherapy was weekly cisplatin $30 \mathrm{mg} / \mathrm{m}^{2}$ (first choice) or cetuximab $250 \mathrm{mg} / \mathrm{m}^{2}$ (cisplatin-ineligible). Patients with T0-2N0-1 disease and no more than 10 pack-years received radiotherapy alone. Treatment response was assessed by positron-emission tomography/computed tomography 10-16 weeks post-CRT. The clinical trials in this study were reviewed and approved by the institutional review board committees. Signed and witnessed consent was obtained from all patients.

Next-generation sequencing (NGS) of tumor samples was performed using UNCSeq [targeted panel-based assay, >200 cancer-associated genes; described previously (11)]. Patients with a more than 10 pack-year history were sequenced using solid tumor mutation panel (Supplementary Table 1, available online).

We estimated disease-free survival (DFS) using KaplanMeier method and log-rank test. Clinical characteristics were compared by $t$ test $/ \chi^{2}$ test; statistically significant $P$ values were less than .05. Cox regression model is described in Supplementary Methods (available online). Formal tests of the proportionality of hazards were not performed because of the number of events, but results were verified based on the Kaplan-Meier DFS curves. All tests were two-sided, and a $P$ value of less than .05 was considered statistically significant.

In this prospective cohort, 77 patients had tumor sequencing. Clinical characteristics are described in Table 1 . Seventyfive patients were treated with 60Gy deescalated CRT; two patients with TP53 mutations received $70 \mathrm{~Gy}$. Median follow-up was 24 months. Of the 77 patients, 34 had mutation(s) identified (16 PIK3CA, 4 PTEN, 3 KRAS, 3 FBXW7, 3 FGFR3, 2 TP53, 1 NRAS, 1 BRCA1, 1 FLT3, 1 RAD50, 1 PIK3R1, 1 EP300; Supplementary Figure 1 and Supplementary Table 1, available online). PIK3CA was the most commonly mutated gene (20.8\%). Of 16 patients, 10 had activating mutations in helical domain (E542K, 8/16, and E545K, 2/16) (12).

TMN staging and tobacco history were similar in patients with wild-type (WT)-PIK3CA and mutated PIK3CA (PIK3CA ${ }^{\text {mut; }}$ Table 1). Disease recurrence patterns in our cohort were similar to previous studies (2): nine patients had recurrence (two regional-only, four distant-only, three regional and distant). One WT-PIK3CA patient died from chemotherapy-related respiratory failure. Overall survival did not differ between the two groups (WT $=97.7 \%, 95 \%$ confidence interval $[\mathrm{CI}]=93.3 \%$ to $100 \%$ vs $\mathrm{PIK} 3 \mathrm{CA}^{\mathrm{mut}}=100 \% ; \mathrm{P}=.67$ ) (Supplementary Figure 2, available online). Five of nine patients with recurrences had known cancer-associated mutations, all of which were PIK3CA mutations. Patients with WT-PIK3CA had statistically significantly higher 3-year DFS $(93.4 \%, 95 \% \mathrm{CI}=85.0 \%$ to 99.9\%) compared with 68.8\% (95\% CI $=26.7 \%$ to $89.8 \%$ ) for PIK3CA ${ }^{\text {mut }}$ patients $(P=.004$; Figure 1$)$. On multivariate analysis

Table 1. Patient characteristics

\begin{tabular}{|c|c|c|c|c|}
\hline Characteristic & $\begin{array}{c}\text { Total } \\
\text { No. }(\%) \\
(\mathrm{N}=77)\end{array}$ & $\begin{array}{c}\text { WT-PIK3CA } \\
\text { No. (\%) } \\
(\mathrm{n}=61)\end{array}$ & $\begin{array}{c}\text { PIK3CA }{ }^{\text {muta }} \\
\text { No. (\%) } \\
(\mathrm{n}=16)\end{array}$ & $P^{*}$ \\
\hline Age, mean (range), y & $60(39-84)$ & $58(39-80)$ & $60(39-84)$ & .53 \\
\hline \multicolumn{5}{|l|}{ Sex } \\
\hline Male & $66(85.7)$ & $52(85.2)$ & $14(87.5)$ & .82 \\
\hline Female & $11(14.3)$ & $9(14.8)$ & $2(12.5)$ & \\
\hline Smoking history & & & & .31 \\
\hline Never & $33(42.9)$ & $28(45.9)$ & $5(31.3)$ & \\
\hline$\leq 10 y$ & $21(27.3)$ & $16(26.2)$ & $5(31.3)$ & \\
\hline$>10 y$ & $23(29.9)$ & $17(27.9)$ & $6(37.5)$ & \\
\hline Tumor stage & & & & .68 \\
\hline T0 & $5(6.5)$ & $4(6.6)$ & $1(6.3)$ & \\
\hline $\mathrm{T} 1$ & $18(23.4)$ & $14(23.0)$ & $4(25.0)$ & \\
\hline $\mathrm{T} 2$ & $50(64.9)$ & 39 (63.9) & $11(68.8)$ & \\
\hline T3 & $4(5.2)$ & $4(6.5)$ & 0 & \\
\hline $\mathrm{T} 4$ & 0 & 0 & 0 & \\
\hline Nodal stage, 7th edition (8th edition) & & & & .32 \\
\hline NO & $6(7.8)$ & $5(8.2)$ & $1(6.3)$ & \\
\hline $\mathrm{N} 1$ & $11(14.3)$ & $10(16.4)$ & $1(6.3)$ & \\
\hline $\mathrm{N} 2 \mathrm{a}(\mathrm{N} 1)$ & $3(3.9)$ & $2(3.3)$ & $1(6.3)$ & \\
\hline $\mathrm{N} 2 \mathrm{~b}(\mathrm{~N} 1)$ & $47(61.0)$ & $37(60.7)$ & $10(62.5)$ & \\
\hline $\mathrm{N} 2 \mathrm{c}(\mathrm{N} 2)$ & $10(13.0)$ & $7(11.5)$ & $3(18.8)$ & \\
\hline N3 & 0 & 0 & 0 & \\
\hline Radiation dose & & & & .46 \\
\hline 60 Gy & $75(97.4)$ & $59(96.7)$ & $16(100.0)$ & \\
\hline 70 Gy & $2(2.6)^{\prime}$ & $2(3.3)$ & 0 & \\
\hline Concurrent chemotherapy/systemic therapy & & & & .76 \\
\hline Yes & $66(85.7)$ & $52(85.2)$ & $14(87.5)$ & \\
\hline No & $11(14.3)$ & $9(14.8)$ & $2(12.5)$ & \\
\hline
\end{tabular}

${ }^{*} \chi^{2}$ test, two-sided $P$ values reported. $\mathrm{WT}=$ wild-type. 


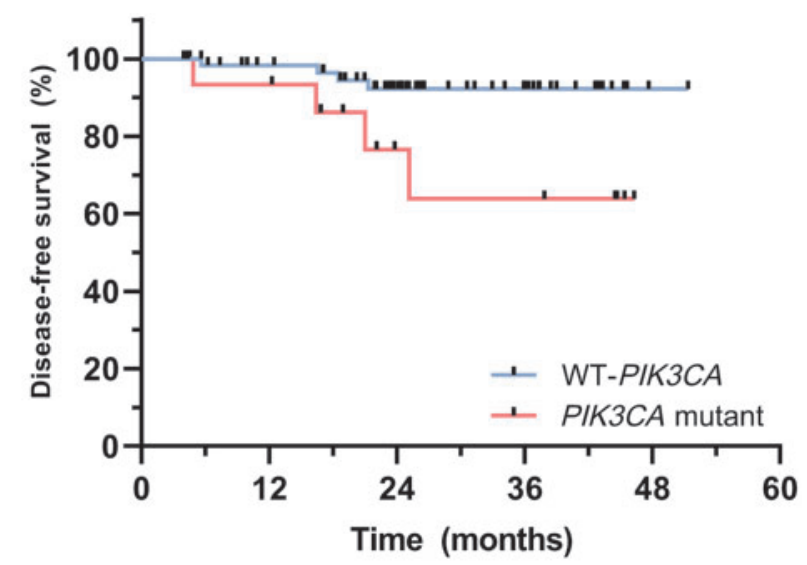

\begin{tabular}{|c|c|c|c|c|}
\hline \multicolumn{5}{|c|}{ Number at risk } \\
\hline WT & 61 & 52 & 35 & 16 \\
\hline Mutar & 16 & 14 & 7 & 6 \\
\hline
\end{tabular}

Figure 1. Disease-free survival in patients with WT-PIK3CA vs PIK3CA mutations Kaplan-Meier estimates of disease-free survival, log-rank test, two-sided $\mathrm{P}=.004$. WT, $\mathrm{n}=61$. PIK3CA mutation, $\mathrm{n}=16$. Median time to recurrence was 17.5 (4.9-25.2) months for WT-PIK3CA and 21.0 (5.6-21.2) months for patients with PIK3CA mutations. WT $=$ wild-type.

accounting for age, gender, smoking, TMN stage, mutation, and receipt of chemotherapy, only PIK3CA mutation was statistically significantly associated with disease recurrence (hazard ratio $=5.71$, $95 \% \mathrm{CI}=1.53$ to $21.3 ; P=.01$ ).

Among the PIK3CA ${ }^{\text {mut }}$ patients with disease recurrence, two had E542K mutations, two E545K mutations, and one E726K mutation. Five PIK3CA ${ }^{\text {mut }}$ patients with disease recurrence received immunotherapy; three had durable responses (14-17 months; Supplementary Table 2, available online).

Seminal previous work demonstrated PIK3CA is commonly mutated in HPV-associated OPSCC patients $(7,9,12,13)$. Here, we present prospectively collected NGS data to study the clinical significance of PIK3CA mutations in this patient population. Consistent with previous studies $(7,14), 20.8 \%$ of patients had PIK3CA mutations. We show that PIK3CA mutation is associated with worse DFS in HPV-associated OPSCC patients treated with deintensified CRT.

Few studies have evaluated the prognostic significance of PIK3CA mutations in OPSCC. Chiosea et al. (15) studied the effect of PIK3CA, PTEN, and HRAS mutations on disease-specific survival (DSS) in a retrospective series of HPV-associated OPSCC patients treated with surgery followed by adjuvant radiation plus or minus chemotherapy. They found no difference in DSS between WT-PIK3CA and PIK3CA ${ }^{\text {mut }}$ patients. There are a number of possible explanations for the apparent differences between their findings and ours. One interesting difference is that many patients in their study received trimodality therapy vs deescalated CRT in our cohort. This suggests that PIK3CA is a predictive biomarker because PIK3CA ${ }^{\text {mut }}$ patients have worse outcomes than WT-PIK3CA patients after deescalated CRT but similar DSS to WT-PIK3CA patients after escalated (trimodality) therapy (9).

In addition, PIK3CA ${ }^{\text {mut }}$ patients with disease recurrences may have been salvaged, resulting in similar long-term DSS to WT-PIK3CA patients (15). It was recently shown that PIK3CA ${ }^{\text {mut }}$ patients with metastatic HPV-positive OPSCC have improved survival (16). Our study does not contradict these findings, as eight of nine patients with recurrent disease remain alive with a median follow-up time of 15 months after recurrence. Interestingly, all three PIK3CA mutant alleles in patients with recurrence (E542K, E545K, E726K) have been implicated as targets of APOBEC mutagenesis and improved responses to immunotherapy in solid tumors (17-19). Consistent with this, three of five PIK3CA ${ }^{\text {mut }}$ patients with recurrence who were treated with immunotherapy experienced durable clinical response in our study. Collectively, these findings suggest that PIK3CA mutations in HPV-associated OPSCC are associated with resistance to deescalated CRT and possibly improved response to immunotherapy.

Although our study has multiple strengths (ie, prospective design), limitations include relatively small size. Nevertheless, differences observed in DFS were highly statistically significant $(P<.01)$. Overall survival was similar between WT- and PIK3CA ${ }^{\text {mut }}$ patients but may differ with longer follow-up. Validation in a larger cohort (eg, National Surgical Adjuvant Breast and Bowel Project (NSABP), the Radiation Therapy Oncology Group (RTOG), and the Gynecologic Oncology Group (GOG)) is an important next step. Furthermore, NGS availability in community practice may limit widespread applicability of the study; however, liquid biopsy may mitigate this.

PIK3CA mutations may be an adverse prognostic factor in HPV-associated OPSCC patients treated with definitive CRT. Patients with PIK3CA mutations may not be suitable for deintensified therapy, and investigation of novel treatment strategies, such as with integration of immune checkpoint blockade therapy, may be appropriate.

\section{Funding}

This work was supported by the Department of Radiation Oncology, University of North Carolina Hospitals and Lineberger Comprehensive Cancer Center, Chapel Hill, North Carolina.

\section{Notes}

Affiliations of authors: Department of Radiation Oncology, University of North Carolina Hospitals, Chapel Hill, NC (BTB, DHM, CJS, NCS, GPG, BSC); Department of Radiation Oncology, University of Florida College of Medicine, Gainesville, FL (RJA, WMM); Department of Hematology/Oncology (JW, JG-O, SP) and Department of Otolaryngology/Head and Neck Surgery (WGY, AZ, TGH, BT, JMB, SNP, MCW, WGY) and Department of Pathology (KEW, LAR) and Department of Genetics (JP) and Department of Biostatistics (XT), University of North Carolina, Chapel Hill, NC; Department of Medical Oncology, University of Tennessee, Memphis, TN (DNH); University of Florida Health Proton Therapy Institute, Jacksonville, FL (RD).

The funders had no role in the design of the study; the collection, analysis, and interpretation of the data; the writing of the manuscript; and the decision to submit the manuscript for publication.

We thank the University of North Carolina Department of Radiation Oncology/Lineberger Comprehensive Cancer Center for their support and for thoughtful feedback on the discussion portion of the manuscript.

BSC holds ownership interest (including patents) in and is a consultant/advisory board member for Naveris. GPG holds ownership interest (including patents) in and is a consultant/advisory board member for Naveris. No potential conflicts of interest were disclosed by the other authors. 


\section{References}

1. Ang KK, Harris J, Wheeler R, et al. Human papillomavirus and survival of patients with oropharyngeal cancer. N Engl J Med. 2010;363(1):24-35.

2. Gillison ML, Trotti AM, Harris J, et al. Radiotherapy plus cetuximab or cisplatin in human papillomavirus-positive oropharyngeal cancer (NRG Oncology RTOG 1016): a randomised, multicentre, non-inferiority trial. Lancet. 2019;393(10166):40-50.

3. Chera BS, Amdur RJ, Tepper J, et al. Phase 2 trial of de-intensified chemoradiation therapy for favorable-risk human papillomavirus-associated oropharyngeal squamous cell carcinoma. Int J Radiat Oncol Biol Phys. 2015;93(5): 976-985.

4. Mehanna H, Robinson M, Hartley A, et al. Radiotherapy plus cisplatin or cetuximab in low-risk human papillomavirus-positive oropharyngeal cancer (De-ESCALaTE HPV): an open-label randomised controlled phase 3 trial. Lancet. 2019;393(10166):51-60.

5. Chera BS, Amdur RJ, Tepper JE, et al. Mature results of a prospective study of deintensified chemoradiotherapy for low-risk human papillomavirusassociated oropharyngeal squamous cell carcinoma. Cancer. 2018;124(11): 2347-2354.

6. de la Rochefordiere A, Kamal M, Floquet A, et al. PIK3CA pathway mutations predictive of poor response following standard radiochemotherapy $+/$ - cetuximab in cervical cancer patients. Clin Cancer Res. 2015;21(11) 2530-2537.

7. Stransky N, Egloff AM, Tward AD, et al. The mutational landscape of head and neck squamous cell carcinoma. Science. 2011;333(6046):1157-1160.

8. Sewell A, Brown B, Biktasova A, et al. Reverse-phase protein array profiling of oropharyngeal cancer and significance of PIK3CA mutations in HPV-associated head and neck cancer. Clin Cancer Res. 2014;20(9): 2300-2311.
9. The Cancer Genome Atlas Network. Comprehensive genomic characterization of head and neck squamous cell carcinomas. Nature. 2015;517(576): 576-582.

10. Amin MB, Edge S, Greene F, et al. AJCC Cancer Staging Manual 8th ed. New York: Springer International Publishing; 2017.

11. Zhao $\mathrm{X}$, Wang A, Walter $\mathrm{V}$, et al. Combined targeted DNA sequencing in nonsmall cell lung cancer (NSCLC) using UNCseq and NGScopy, and RNA sequencing using UNCqeR for the detection of genetic aberrations in NSCLC. PLoS One. 2015;10(6):e0129280.

12. Nichols AC, Palma DA, Chow W, et al. High frequency of activating PIK3CA mutations in human papillomavirus-positive oropharyngeal cancer. JAMA Otolaryngol Head Neck Surg. 2013;139(6):617-622.

13. Bratman SV, Bruce JP, O'Sullivan B, et al. Human papillomavirus genotype association with survival in head and neck squamous cell carcinoma. JAMA Oncol. 2016;2(6):823-826

14. Janku F, Yap TA, Meric-Bernstam F. Targeting the PI3K pathway in cancer: Are we making headway? Nat Rev Clin Oncol. 2018;15(5):273-291.

15. Chiosea SI, Grandis JR, Lui VWY, et al. PIK3CA, HRAS and PTEN in human papillomavirus positive oropharyngeal squamous cell carcinoma. BMC Cancer. 2013;13(1):602

16. Hanna GJ, Kacew A, Chau NG, et al. Improved outcomes in PI3K-pathwayaltered metastatic HPV oropharyngeal cancer. JCI Insight. 2018;3(17):1-11.

17. Gillison ML, Akagi K, Xiao W, et al. Human papillomavirus and the landscape of secondary genetic alterations in oral cancers. Genome Res. 2019;29(1):1-17.

18. Henderson S, Chakravarthy A, Su X, et al. APOBEC-mediated cytosine deamination links PIK3CA helical domain mutations to human papillomavirusdriven tumor development. Cell Rep. 2014;7(6):1833-1841.

19. Miao D, Margolis CA, Vokes NI, et al. Genomic correlates of response to immune checkpoint blockade in microsatellite-stable solid tumors. Nat Genet. 2018;50(9):1271-1281. 